\title{
Fast Radio Burst Dispersion Measure Distribution as a Probe of Helium Reionization
}

\author{
Mukul Bhattacharya ${ }^{1,2}$, Pawan Kumar ${ }^{3}$, Eric V. Linder ${ }^{4,5}$, \\ ${ }^{1}$ Center for Neutrino Physics, Department of Physics, Virginia Tech, Blacksburg, VA 24061, USA \\ ${ }^{2}$ Department of Physics; Department of Astronomy 83 Astrophysics; Center for Multimessenger Astrophysics, \\ Institute for Gravitation and the Cosmos, The Pennsylvania State University, University Park, PA 16802, USA \\ ${ }^{3}$ Department of Astronomy, University of Texas, Austin, TX 78712, USA \\ ${ }^{4}$ Berkeley Center for Cosmological Physics 8 Berkeley Lab, \\ University of California, Berkeley, CA 94720, USA \\ ${ }^{5}$ Energetic Cosmos Laboratory, Nazarbayev University, Nur-Sultan 010000, Kazakhstan
}

(Dated: October 29, 2020)

\begin{abstract}
Fast radio burst (FRB) discoveries are occurring rapidly, with thousands expected from upcoming surveys. The dispersion measures (DM) observed for FRB include important information on cosmological distances and the ionization state of the universe from the redshift of emission until today. Rather than considering the DM-redshift relation, we investigate the statistical ensemble of the distribution of dispersion measures. We explore the use of this abundance information, with and without redshift information, to probe helium reionization. Carrying out Monte Carlo simulations of FRB survey samples, we examine the effect of different source redshift distributions, host galaxy models, sudden vs gradual reionization, and covariance with cosmological parameters on determination of helium reionization properties. We find that a fluence limited survey with $10^{4} \mathrm{FRBs}$ can discriminate different helium reionization histories at $\sim 6 \sigma$ using the DM-distribution of bursts, without redshift information (and $\sim 10 \sigma$ with redshifts).
\end{abstract}

\section{INTRODUCTION}

The energetic millisecond duration transients known as fast radio bursts are fascinating in themselves, for their potential insights into compact objects, magnetic fields and plasmas, and particle acceleration [1-14]; see [15] for a recent review. They also serve as bright backlights to the intergalactic medium (IGM), visible to redshifts $z>1$, and providing dispersion measures containing information on the distance along the line of sight, and the electron density and hence ionization state of the intervening universe.

Thousands of FRB and their DM will be detected by currently ongoing and upcoming radio surveys, potentially out to $z \gg 1[16-20]$. A smaller subset will also have host galaxy localization and associated redshift information. Numerous articles have already considered using them as probes to study the dark energy equationof-state from cosmic distance measures (see, e.g., [2125]), while others have addressed their use as ionization measures, particularly seeking to detect and characterize He II reionization at $z_{r} \approx 3$ (see, e.g., [26, 27]). For a general review of FRB literature, see [28, 29].

The strongest observational evidence of He reionisation comes from the far ultraviolet spectra of the HeII Ly $\alpha$ forest along the sightlines of multiple quasars that extend up to $z \sim 3.8[30,31]$. In particular, the average effective HeII Ly $\alpha$ optical depth evolves from $\tau_{\text {eff }} \sim 2$ at $2.7 \lesssim z \lesssim 2.9$ to $\tau_{\text {eff }} \sim 5$ at $3.2 \lesssim z \lesssim 3.6$. Although the lower redshift of He reionisation (compared to hydrogen reionisation) makes it more accessible for detailed studies, the comparatively fewer number of sightlines that exhibit the Ly $\alpha$ forest signature places a high statistical uncertainty on the precise timing and nature of the reionisation process. An alternate method to identify the He reionisation epoch arises from the cosmological simulations that have studied the evolution of IGM temperature around $z \sim 3$ [30].

Large FRB samples will, at least initially, largely lack redshift information. One approach is to turn to the ensemble properties of the bursts, such as their abundance as a function of DM (see, e.g., [32-35]). This too encodes information on helium reionization and will be the main focus of this article. We explore the relation between measured distributions $d n / d D M$ and $d n / d z$ to learn about the redshift of helium reionization.

In Section II we describe how we can learn about the helium reionization epoch from the shape of FRB DMdistribution; we note that measurement of redshifts of FRBs will be available only for a small sample of bursts whereas the entire sample of FRBs in any survey can be used to construct the DM-distribution. We discuss an analytic probabilistic approach to relate the observed DM distribution $d n / d D M$ to the source redshift distribution $d n / d z$, as well as Monte Carlo simulations for "inverting" the FRB DM-distribution to obtain evidence for He-reionization. We carry out the probabilistic approach in Section III to compute in Section IV the statistical significance expected for a detection of helium reionization. Turning to Monte Carlo simulations in Section V, we describe our set up including different source distribution and host galaxy models. Section VI presents the results using $d n / d z$ directly, while Section VII applies the approach with $d n / d D M$. We conclude in Section VIII.

\section{ABUNDANCE DISTRIBUTION}

Helium II reionization injects additional electrons into the IGM, raising the electron density and increasing the 
DM per unit path length. This is approximately a $7 \%$ effect, and should occur some time between redshift 6 (approximately when hydrogen and Helium I ionization occurs) and redshift 3 . Initially we consider it to be a sudden event, at redshift $z_{r}$. Since it changes DM, this will impart a feature to the abundance distribution $d n / d D M$, the number of FRB per unit interval of DM.

This impact on abundance, i.e. the ensemble statistics of FRB rather than the effect on any individual burst or set of bursts at a given redshift, is the focus of this section. In particular, since for every detected FRB we obtain a DM measurement, but for relatively few we measure host galaxy redshift (since localizing the burst is not trivial, and the burst itself does not clearly indicate redshift), the statistics in terms of DM is much larger than in terms of redshift.

We present a pedagogical discussion of alternative methods to relate $d n / d D M$ and $d n / d z$ before proceeding with our chosen approach in Sec. III.

\section{A. Shape Approach}

The most conceptually straightforward approach in principle is simply to study the shape of $d n / d D M$, looking for a bend in the curve indicating a modification in the DM function from the $\sim 7 \%$ change in the ionization fraction due to helium reionization. If this is a sudden transition then the bend will be a kink in the curve. However, we must realize that it is not a break in the slope in the sense that above and below the reionization event the relation is not linear - in general there will be some curvature both above and below and we will need to recognize the bend. In practice this involves fitting the distribution for a range on either side, and so does not offer practical advantages over using the full distribution. If one does identify the bend, this merely says that something happens at that DM; we would still have to propagate that information to redshift, e.g. using the homogeneous cosmology relation $D M(z)$, if we want the reionization redshift.

\section{B. Direct Approach}

One could also use the more limited information one has on $d n / d z$, for those FRB with redshifts. One could take the observational dataset of $d n / d z$ and the dataset of $d n / d D M$ and simply form

$$
\frac{d D M}{d z}=\frac{d n / d z}{d n / d D M}
$$

This can then be related to reionization since for the IGM (cosmological) component,

$$
\frac{d D M}{d z}=\frac{1+z}{H(z) / H_{0}} n_{e, 0} f_{e}(z)
$$

where $n_{e, 0}$ is the electron density today and $f_{e}$ is the electron (or baryon) fraction relative to the homogeneous, fully ionized, fully hydrogen state. Again we look for a marked change in $f_{e}(z)$.

An advantage of the direct approach is that because we are taking ratios of abundances it is possible that common systematics due to selection functions may cancel out.

However, one major problem with the direct approach is that we have to match the DM bin with the $z$ bin. One might perhaps be able to do this through claiming that with large number statistics the homogeneous relation $D M(z)$ holds, but this is not assured, especially with a second issue of an uncertain host galaxy contribution to subtract off from the observed DM to obtain the IGM component. The uncertainty and the bias of the actually realized relation for the sample would have to be accounted for.

\section{Probabilistic Approach}

The direct approach relies on perfect (or perfectly averaged) homogeneity. But the problem is similar to that for photometric vs spectroscopic surveys in optical astronomy. The measured, photometric redshift is not a perfect tracer of the true, spectroscopic redshift. Instead one must integrate over the probability distribution connecting the two. For our case, this would be

$$
\frac{d \tilde{n}}{d z}=\int d D M p(z \mid D M) \frac{d n}{d D M}
$$

where now $d \tilde{n} / d z$ is the derived, not measured distribution, and $p(z \mid D M)$ is the probability that the measured DM corresponds to some redshift $z$. This derived distribution $d \tilde{n} / d z$ could then be compared to the observed $d n / d z$ to look for agreement. The form $p(z \mid D M)$ could be adjusted until it achieves this, and in particular one could compare the results for a $p(z \mid D M)$ that did not have reionization within the observed redshift range to one that included it at a certain redshift to find a signature of reionization.

Note the same process can be done the other way, with

$$
\frac{d \tilde{n}}{d D M}=\int d z p(D M \mid z) \frac{d n}{d z}
$$

and one can use Bayes' Theorem as a crosscheck,

$$
p(D M \mid z)=\frac{p(z \mid D M) p(D M)}{p(z)} .
$$

Finally, one can again form the ratio in Eq. (1) to redo the direct approach with the probabilistic expressions and obtain

$$
\frac{d D M}{d z}=\frac{\int d D M p(z \mid D M) d n / d D M}{d n / d D M} .
$$




\section{CALCULATING WITH THE PROBABILISTIC APPROACH}

The second version of the probabilistic approach, where we compute

$$
\frac{d \tilde{n}(D M)}{d D M}=\int d z p(D M \mid z) \frac{d n}{d z},
$$

has several advantages. We decide to use this for three reasons: 1) the expressions are clearer and it is more intuitive to use $p(D M \mid z), 2)$ the expressions are more Gaussian, and hence easier to use, assuming Gaussian fluctuations in DM due to an inhomogeneous IGM, plus contributions due to a $D M_{\text {host }}$, and 3) in the $\chi^{2}$ comparison that will be the final step the statistics are improved by comparing to the observed $d n / d D M$ rather than the less numerous observed FRB with redshifts entering $d n / d z$.

The two main initial ingredients are the FRB source distribution with redshift, $d n / d z$, and the conditional probability $p(D M \mid z)$. The integral will simply be a sum over bins in $z$. As a first step we take the conditional probability to be given by a Gaussian,

$$
p(D M \mid z) \sim e^{-\left[D M-D M_{\mathrm{He}}(z)\right]^{2} /\left[2 \sigma(z)^{2}\right]} .
$$

Here, DM is the value DM at which the left hand side $d \tilde{n} / d D M(D M)$ of Eq. (7) is evaluated, $D M_{\mathrm{He}}(z)$ is a model evaluated at $z$, and $\sigma^{2}(z)$ is the variance of the Gaussian. The probability integrated over redshift is normalized to unity.

If we take the infinitely sharp limit of the Gaussian probability, we get a delta function. Using the relation $\left.\delta[g(z)]=\delta\left(z-z_{\star}\right) / \mid d g / d z_{\star}\right) \mid$, we find the limit of Eq. (7) to be simply $d \tilde{n} / d D M=(d n / d z) /(d D M / d z)=$ $d n / d D M$, where DM is evaluated at the exact value corresponding to $z$, as given in the $D M(z)$ model. Thus $p(D M \mid z)$ is indeed a kernel, or smearing function.

The $D M(z)$ model is that of Eq. (4) of [27], taking into account reionization at $z_{r}$,

$$
D M_{\mathrm{He}}(z)=D M(z)_{\mathrm{high}}+A_{H e}\left[D M(z)_{z_{r}}-D M(z)_{\mathrm{high}}\right]
$$

where the subscript "high" means reionization occurs beyond the limits of the sample, e.g. $z>5$, while the subscript " $z_{r}$ " means it occurs at $z=z_{r}{ }^{1}$. The amplitude $A_{H e}$ measures the strength of the reionization, with $A_{H e}=0$ saying reionization is not present within

\footnotetext{
1 To distinguish clearly this analytic probabilistic approach from the Monte Carlo simulation approach of later sections, we intentionally choose different forms and parameter values; for example, there is no need for $A_{H e}$ in the Monte Carlo simulations since the reionization dependence goes into the simulation. The commonality is in the concept; results should not be directly compared.
}

the sample range and $A_{H e}=1$ means it has the fiducial strength. The dispersion measure is the usual

$$
D M(z)=H_{0}^{-1} n_{e, 0} \int_{z_{e}}^{z_{o}} \frac{d z(1+z)}{H(z) / H_{0}} f_{e}(z),
$$

where

$$
f_{e}(z)=(1-Y) f_{H}+\frac{Y}{4}\left(f_{H e I I}+2 f_{H e I I I}\right),
$$

so that $f_{e}\left(z>z_{r}\right)=1-3 Y / 4 \approx 0.818$ and $f_{e}\left(z<z_{r}\right)=$ $1-Y / 2 \approx 0.879$, where $z_{r}$ is the redshift of (sudden) reionization. (Also see Appendix A.)

We can take the standard deviation $\sigma(z)$ to be given by the inhomogeneous IGM fluctuations (ignoring host contributions for now),

$$
\sigma(z)=210 \sqrt{z}
$$

in good agreement with simulations [36] (see Sec. V B for further discussion) and following [25, 27].

Once we carry out the integral in Eq. (7) by summing over redshift bins, we compare the result $d \tilde{n} / d D M$ to the measured $d n / d D M$. This comparison is quantified with

$$
\chi^{2}=\sum_{D M}\left[\frac{d \tilde{n} / d D M-d n / d D M}{\sigma(d n / d D M)}\right]^{2},
$$

where the sum is over bins of DM. The uncertainty $\sigma(d n / d D M)$ here is just the Poisson fluctuation of the numbers in each DM bin, $\sigma(d n / d D M)=\sqrt{d n / d D M}$.

What we are interested in is the variation of the $\chi^{2}$ as we change the reionization characteristics. That is, does $A_{H e}=1$ give a better fit than $A_{H e}=0$, say? We can map out the $\chi^{2}$ surface for variations in $A_{H e}$ and $z_{r}$.

Since sufficient actual FRB data extending beyond the likely reionization redshift does not yet exist, we have to simulate it. As a first step we will use the distribution suggested in Eq. (6) of [27],

$$
\frac{d n}{d z}=\frac{N_{\text {tot }}}{N_{\text {norm }}} z^{3} e^{-z / z_{\star}}
$$

where $N_{\text {norm }}=z_{\star}^{4}\left[6-e^{-y}\left(y^{3}+3 y^{2}+6 y+6\right)\right]$, with $y=z_{\max } / z_{\star}$, is a normalization constant to give $N_{\text {tot }}$ total FRB with redshifts $z<z_{\max }$. The distribution peaks at $3 z_{\star}$, and we might choose $z_{\star}=1$. Similarly we can simulate the $d n / d D M$ distribution with $A_{H e}=1$ and $z_{r}=3$. Initially we will take the distributions as stated; later we will add Poisson fluctuations in the realizations.

\section{ANALYTIC ABUNDANCE RESULTS}

With all the ingredients in place needed for Eq. (7), we carry out the calculations. Figure 1 shows $p(D M \mid z)$ for several values of redshift. For completeness, Figure 2 shows $p(z \mid D M)$, though we do not use this quantity. Using the distribution $p(D M \mid z)$, we then compute $d \tilde{n} / d D M$ 
for $N_{\text {tot }}=500$, shown in Figure 3 for standard reionization at $z_{r}=3$ (labeled as $A_{H e}=1$ ), and for no reionization in range $\left(A_{H e}=0\right)$. It is the difference between these two curves, shown as a percent variation by the dotted red curve, that allows distinction between the reionization and no reionization scenarios given data.

We quantify this by evaluating Eq. (13) to compute the $\chi^{2}$ difference between the two cases delivered by the mock data. That is, if $d \tilde{n} / d D M$ arises assuming that $p(D M \mid z)$ is given with no reionization during the redshift range of observation, we compute how well this can be tested by observations of $d n / d D M$ that occur in a universe that does have reionization at $z_{r}=3$. As a first estimate we take $d n / d z$ to be exactly given by Eq. (14); later we will include Poisson fluctuations in its realization. Poisson fluctuations in $d n / d D M$ are accounted for in the denominator of Eq. (13).

Figure 4 shows the $\Delta \chi^{2}$ results. The no reionization $\left(z_{r}>5\right)$ model can be distinguished from the $z_{r}=3$ reionization model by $\Delta \chi^{2}=17.3$, or somewhat over $4 \sigma$. From the instantaneous $\Delta \chi^{2}$ curve (i.e. the contribution to $\Delta \chi^{2}$ from each interval of $\mathrm{DM}$ ) we see that FRB lying well above the reionization redshift (at around $\mathrm{DM} \approx 2900$ ) have the greatest leverage. This accords with Fig. 3, where the difference between the two models comes from the rapidly declining upper edge to the distribution. One will have to be careful of selection effects to make sure these do not bias the results. By $\mathrm{DM} \approx 4300$ one has almost the full signal for distinguishing the models since there are very few observed FRB for larger DM - recall that our $d n / d z$ here has an exponential cutoff and we take the survey depth to be $z<5$.

Including Poisson fluctuations in the realization of the observed $d n / d D M$ we obtain Figure 5 . Here we show the $\chi^{2}$ for the standard reionization $\left(A_{H e}=1\right)$ model vs its realization and the no reionization $\left(A_{H e}=0\right)$ model vs the standard model realization. For the standard model, the $\chi^{2}(D M)$ per DM bin simply scatters about 1 , so the summed $\chi^{2}$ increases roughly linearly (dashed curves). On the other hand, for the no reionization model (solid curves) there is a clear signature of a deviation peaking around $D M=4100$, unmatched by the dashed curve, as for the previous exactly realized case. With the realization scatter, the standard model is preferred by $\Delta \chi^{2} \approx-8$ over the no reionization model, somewhat less than $3 \sigma$. Note that the total $\chi^{2}$ for the standard model vs its realization is somewhat less than 50 (the number of bins) because for bins with very few FRB the $\sqrt{N}$ fluctuations used (rather than true Poisson statistics) overestimate the error and so gives overly small $\chi^{2}$. However, this should have little effect on the difference $\Delta \chi^{2}$ between the standard and no reionization models.

Thus we have given an analytic view of how the FRB DM distribution can distinguish between reionization models. In the remainder of the article we turn to Monte Carlo simulations to quantify this more robustly and test the dependence on the astrophysical ingredients going

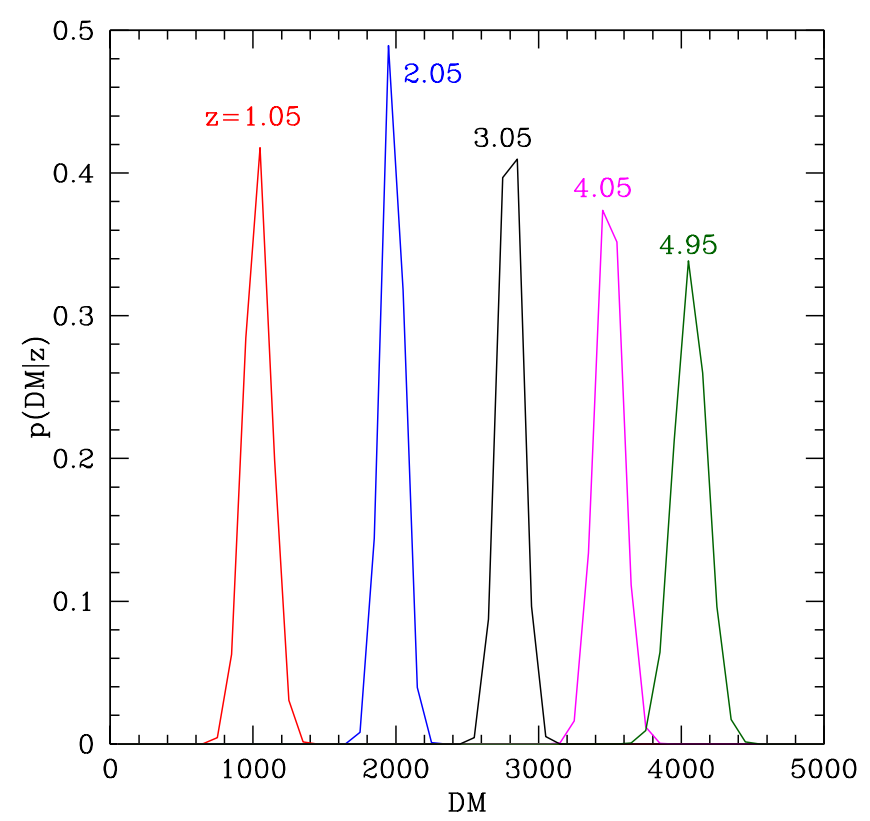

FIG. 1. The conditional probability $p(D M \mid z)$, the probability to measure a value DM given a (possibly unknown) true redshift $z$, is shown for five values of $z$. The DM values represent the cosmological contribution including inhomogeneous IGM; host DM contributions are not included.

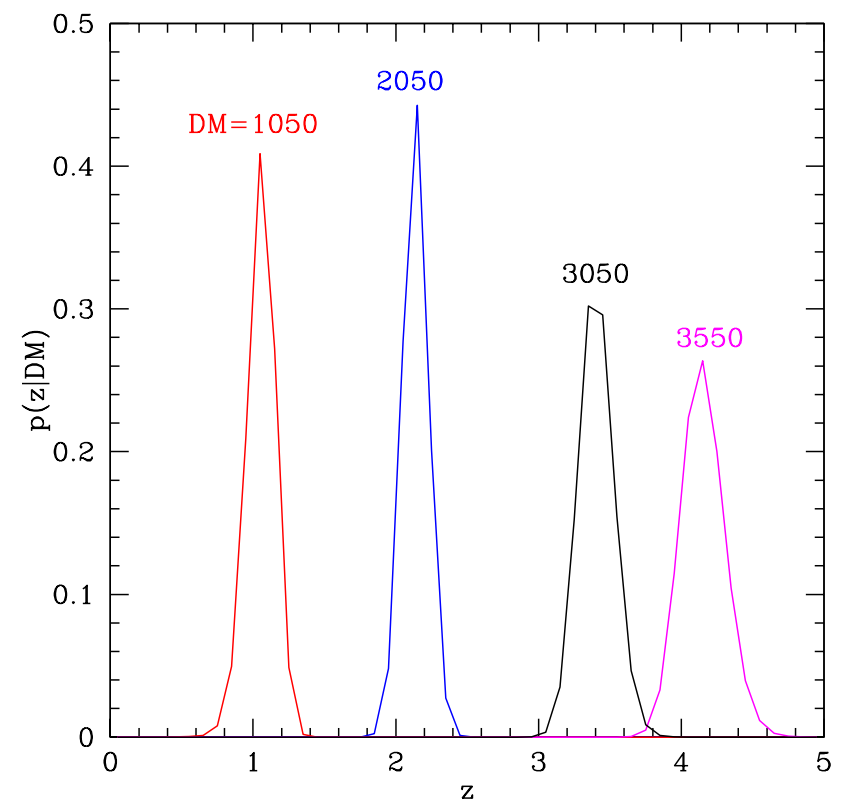

FIG. 2. As Fig. 1, but for the conditional probability $p(z \mid D M)$, the probability to assign a redshift $z$ given a measured DM, is shown for four values of DM. This quantity is not used in the calculations. 


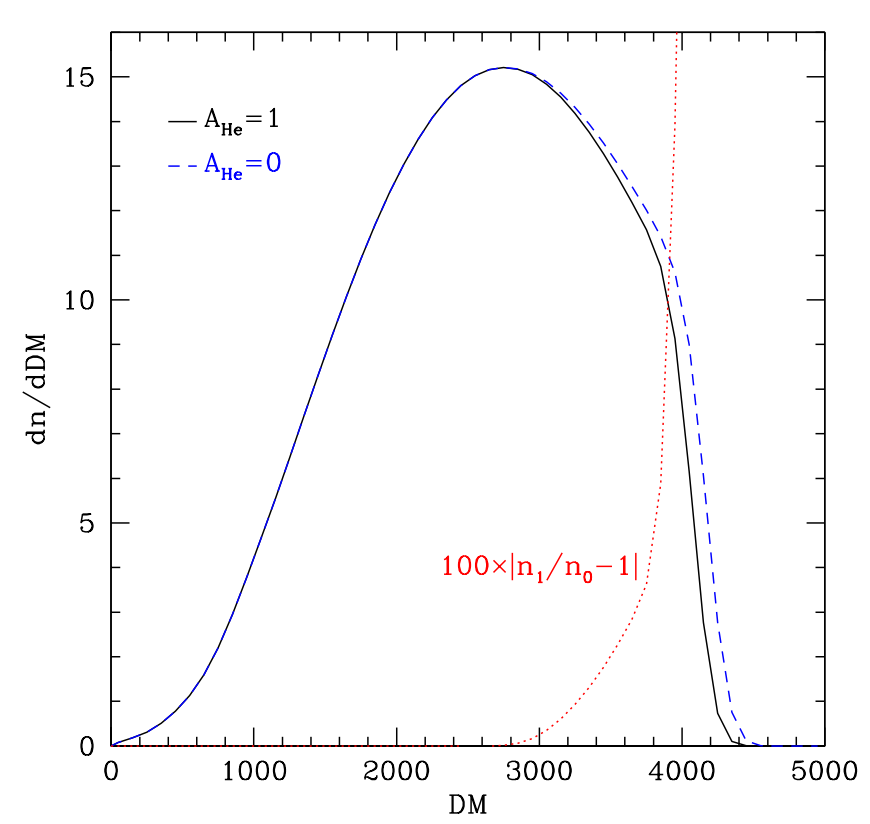

FIG. 3. The distribution of the number of FRB per unit $\mathrm{DM}, d n / d D M$, is plotted vs DM. The solid black curve shows the fiducial case of standard helium reionization at $z_{r}=3$ $\left(A_{H e}=1\right)$, while the dashed blue curve has no helium reionization within observed range $\left(A_{H e}=0\right.$, i.e. $\left.z_{r}>5\right)$. The percent difference between the two curves is given by the dotted red curve, showing a $6 \%$ difference at $\mathrm{DM}=3850,14 \%$ at $\mathrm{DM}=3950$, and $32 \%$ at $\mathrm{DM}=4050$. The turnover in both curves is due to the declining assumed population $d n / d z$ beyond $z=3$.

into the observed abundance distribution.

\section{MONTE CARLO SIMULATION APPROACH}

The mostly analytic results of the previous sections give a good indication of the level of distinction one expects in determining the helium reionization redshift through abundance distributions. However we would like to have more sophisticated treatment of several elements. Rather than adding elaborations to the analytic probabilistic approach, we instead incorporate them into Monte Carlo (MC) simulations. Three areas of improvement are: 1) We include the contribution of the host galaxy and local FRB environment to DM and its uncertainties, 2) We study different models for the distribution $n(z)$ and evaluate its scatter in a Monte Carlo manner rather than as $\sqrt{d n / d z}, 3)$ We investigate the impact of the cosmological background, particularly the uncertain value of the matter density $\Omega_{m}$ in a $\Lambda \mathrm{CDM}$ model, on distinction between different reionization models.

We begin by considering redshifts to be known for every FRB, i.e. dealing exclusively with the distribution $d n / d z$. In Sec. VII we combine the probabilistic approach

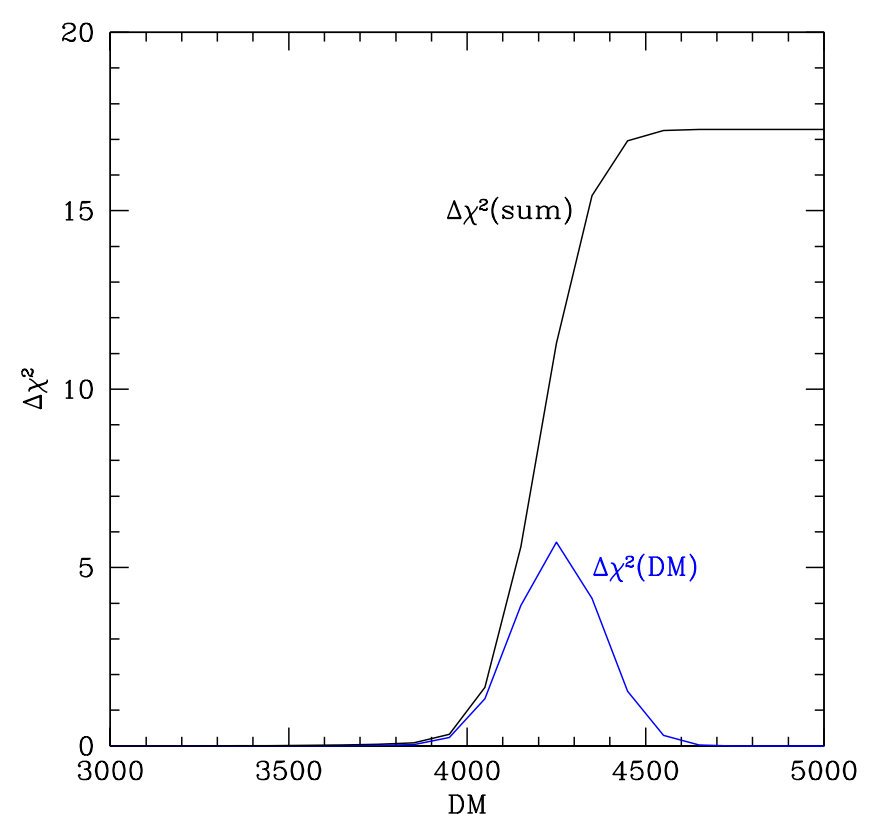

FIG. 4. Distinction between the standard reionization model and a no reionization model can be quantified with $\Delta \chi^{2}$. The lower, blue curve shows the $\Delta \chi^{2}$ contribution from each DM bin, while the upper, black curve shows the cumulative $\Delta \chi^{2}$ from observations out to that DM.

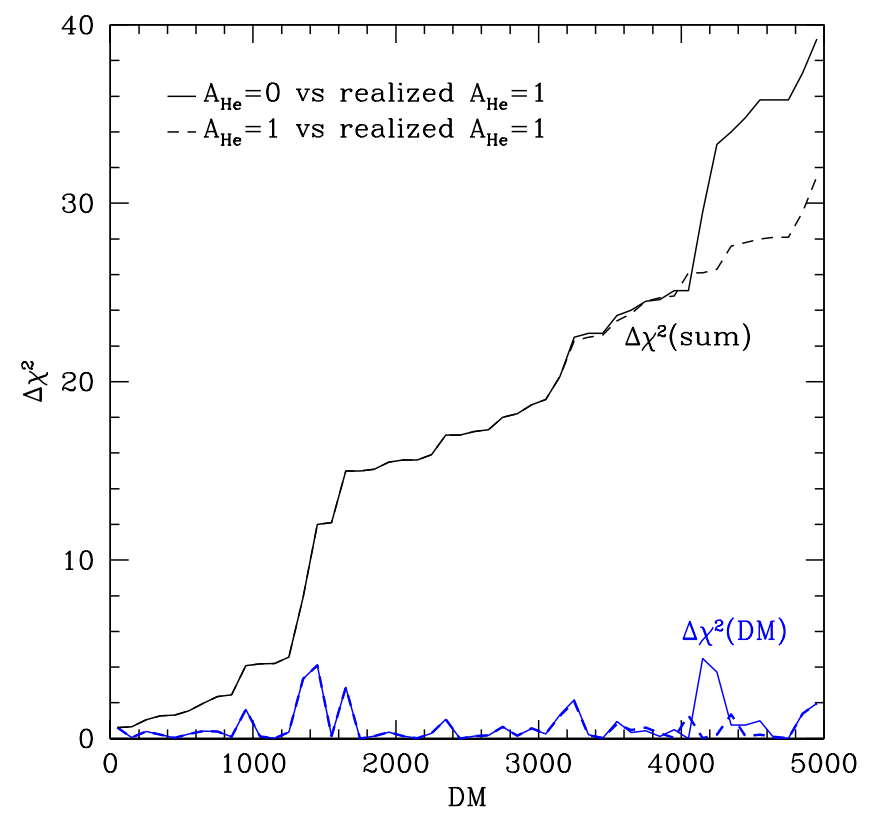

FIG. 5. As Fig. 4 but including realization scatter in the observed $d n / d D M$, taken to be from the standard reionization $\left(A_{H e}=1\right)$ model. The solid curve shows $\Delta \chi^{2}$ for the no reionization $\left(A_{H e}=0\right)$ model relative to the realized data, while the dashed curve shows $\Delta \chi^{2}$ for the standard reionization model relative to its realization (i.e. just random scatter). 
and Monte Carlo to study $d n / d D M$.

For a more complete investigation we consider several variations of the main ingredients entering the observed $D M$ - the redshift distribution and the host and local $D M$ model - and the dependence of the results on the number of FRB observed.

\section{A. FRB Redshift Distribution}

For the redshift distributions $n(z)$ from which we will draw FRB in the simulations we investigate three models: 1) FRB population tracking the cosmic star formation rate (SFR) - this will be our fiducial, 2) Non-evolving (NE) population, i.e. tracing the cosmic volume element, and 3) Constant spatial density (Cons).

For young stellar FRB progenitors, the spatial distribution of FRBs is expected to closely trace the cosmic SFR. We consider the cosmic SFR function

$$
\psi(z)=0.015 \frac{(1+z)^{2.7}}{1+[(1+z) / 2.9]^{5.6}} \mathrm{M}_{\odot} \mathrm{yr}^{-1} \mathrm{Mpc}^{-3},
$$

as proposed by Madau \& Dickinson [37]. The appropriately weighted redshift distribution is obtained by considering the quantity

$$
\zeta_{\mathrm{SFR}}=\frac{\int_{0}^{z} \psi\left(z^{\prime}\right) d z^{\prime}}{\int_{0}^{z_{\max }} \psi\left(z^{\prime}\right) d z^{\prime}},
$$

and drawing it as a uniform random number between 0 and 1 . We take $z_{\max }=6$. The FRB redshifts are then generated by inverting this for the redshift, with a reasonable fit given by

$$
\begin{aligned}
z \approx & 15.05 \zeta_{\mathrm{SFR}}-69.93 \zeta_{\mathrm{SFR}}^{2}+193.7 \zeta_{\mathrm{SFR}}^{3}-271.5 \zeta_{\mathrm{SFR}}^{4} \\
& +184.5 \zeta_{\mathrm{SFR}}^{5}-45.88 \zeta_{\mathrm{SFR}}^{6}
\end{aligned}
$$

For the NE case the number of FRB sources is directly proportional to the comoving volume. Here we draw a random number $\zeta_{\text {vol }}$ between 0 and 1 and assign comoving distances in the flat universe by

$$
D_{c}=\left(\frac{3 \zeta_{\mathrm{vol}} V_{c, \max }}{4 \pi}\right)^{1 / 3}
$$

For the fiducial cosmology of a flat $\Lambda \mathrm{CDM}$ universe with present matter density fraction $\Omega_{m}=0.315$ and a Hubble constant $H_{0}=67.4 \mathrm{~km} / \mathrm{s} / \mathrm{Mpc}$, with $z_{\max }=6$ then $V_{c, \max }=2383 \mathrm{Gpc}^{3}$. The FRB redshift is obtained by inverting $D_{c}=\int_{0}^{z} d z^{\prime} / H\left(z^{\prime}\right)$, where $H(z)=$ $H_{0}\left[\Omega_{m}(1+z)^{3}+1-\Omega_{m}\right]^{1 / 2}$ is the Hubble parameter.

For the constant spatial density model the FRB distribution $n(z)$ is independent of redshift. We normalize all three models to have the same total number of FRB, $N_{\mathrm{FRB}}$, between $z=0-z_{\max }$.

\section{B. Dispersion Measure Components}

Once a FRB is drawn from the $n(z)$ distribution, we model the component contributions to its DM. The total observed FRB DM can be written as

$$
D M_{\mathrm{tot}}=D M_{\mathrm{MW}}+D M_{\mathrm{cos}}+\frac{D M_{\mathrm{host}}}{(1+z)} .
$$

We discuss each of these components - from our Milky Way galaxy, the cosmological propagation through the intergalactic medium (IGM), and host galaxy contribution including the local, or near source, electron density. We describe below how each of these components are modelled in our MC simulations.

Milky Way Galactic contribution: The free electron density varies along different lines of sight within the Milky Way. The NE2001 [38] model uses Galactic pulsars to map the DM contribution from the Milky Way interstellar medium (ISM) along any given FRB sightline. The Galactic ISM $D M_{\mathrm{MW}}$ strongly decreases as a function of Galactic latitude $b$ from $\sim 10^{3} \mathrm{pc} \mathrm{cm}^{-3}$ near the Galactic center to an average of $\sim 10^{2} \mathrm{pc} \mathrm{cm}^{-3}$ at $10<b<40$. As the electron density in the Galactic halo is relatively low with a correspondingly small DM contribution $\sim 30 \mathrm{pc} \mathrm{cm}^{-3}$ suggested from simulations [39], we do not include the extra halo contribution. Our simulations use the NE2001 model value along a randomly generated FRB sightline.

Host galaxy and near-source contributions: The host galaxy DM contribution $D M_{\text {host }}$ arises from its ISM and the environment near the FRB source. Both are highly uncertain. The host ISM contribution depends on the type of host galaxy, galaxy redshift, inclination angle of the galactic disk relative to our sightline, and the site of the FRB source within its galaxy. The near-source plasma contribution can depend on the FRB formation mechanism and the structure of local environment. Furthermore, the evolution of a FRB host galaxy with redshift might lead to the evolution of the host galaxy DM ISM component, also depending on the host galaxy morphology, metallicity, mass, and star-formation rates.

Considerable uncertainty exists concerning these contributions as at the time of manuscript preparation only 9 FRB hosts are listed in FRBCAT [40]. Due to all the galaxy and source uncertainties mentioned above, simulations (e.g. [41-44]) or a small catalog of FRB host galaxies have provided useful, but limited, insight due to observational selection effects associated with host galaxy identification, and how complex gas and radiation processes are handled in simulations. Therefore, we choose three models for the contribution of the FRB host galaxy and its circumgalactic medium (CGM) to the observed DM ( $\left.D M_{\text {host }}\right)$ that span a wide range of parameters, to determine the robustness of the FRB DM-distribution technique to investigate helium reionization history.

We choose as the baseline model a host+local contribution to DM following a Gaussian with mean $270 \mathrm{pc} \mathrm{cm}-3$ and standard deviation $135 \mathrm{pc} \mathrm{cm}^{-3}$, and then consider 
"low" and "high" versions with the low version tracing MW DM (see below) and the high version having twice the baseline Gaussian mean and standard deviation, i.e. $\mathcal{N}(540,270)$. This should cover reasonable cases for $D M_{\text {host }}$. The baseline model is similar to that of [42]. Any Monte Carlo draws from the Gaussian that give negative DM are resampled. Another possible model would be a lognormal distribution (which of course does not give negative DM), however we found that the long tail to higher DM meant that the mean DM was often considerably higher than the mode.

High values seem at odds with most of the cases of FRBs that have actually been localized to a host galaxy, listed in [40]. Subtracting the MW and mean IGM contributions for their measured redshifts, the $9 D M_{\text {host }}$ values (in $\mathrm{pc} \mathrm{cm}^{-3}$ ) for these range from -160 (presumably indicating a severely underdense IGM along that line of sight) to 200, with a mean of 40 . Our low model is closer to this (for a homogeneous IGM). It tracks the MW DM distribution, choosing a random line of sight through the NE2001 map of the electron densities within the MW ISM. Interestingly, the FIRE simulations ([44], also private communication, $\mathrm{X}$. Ma) show some similarity to this. The high model picks up some of the high values that a lognormal would have, and serves as a particularly conservative case for estimation of detecting helium reionization. Thus these three models seem to span a useful range. We further add to $D M_{\text {host }}$ a circumgalactic medium contribution of $D M=65 \pm 15 \mathrm{pc} \mathrm{cm}^{-3}$ [45].

A note regarding the near source contribution included in $D M_{\text {host }}$ above: it is reasonable to assume that a significant portion could actually arise from the nearsource plasma that could be a pulsar wind nebulae, supernova remnant, or HII region [32, 46]. However, this is physically restricted by the fact that the plasma frequency must not exceed the radiation frequency thereby allowing free radio wave propagation across cosmological distances. Most electrons within galaxies are produced when UV radiation emitted by newly-formed massive stars ionises surrounding clouds of gas, also known as HII regions. Galaxies can have a higher abundance of HII regions at larger redshifts as the gas density and star-formation rate density in galaxies increases with redshift. Here we assume that a significant portion of FRB sources are associated with local high density actively star-forming HII regions that can enhance $D M_{\text {host }}$. The distribution of electrons within a galaxy is closely related to the distribution of HII regions, which are mostly found in the arms of spiral galaxies but rarely in dwarf or elliptical galaxies [47].

Intergalactic medium contribution: The $D M_{\mathrm{IGM}}$ contribution for FRBs at similar redshifts but different sightlines can vary considerably due to the fluctuations in electron number density. This is essentially determined by the inhomogeneity of ionized matter in the IGM and halos of intervening galaxies. The sightline to sightline variation from the mean $D M_{\mathrm{IGM}}$ is sensitive to the radial gas profile of the halos as well as the spatial distribution of halos [48]. In particular, the halo models in which the baryon distribution closely tracks the dark matter density profile results in the largest dispersion $\sigma_{\mathrm{IGM}}$ in the $D M_{\text {IGM }}$ component [36].

Using cosmological simulations to model baryonic distribution, [36] obtained results well fit by $\sigma_{\text {IGM }} \approx 210 \sqrt{z}$. Further recent simulations bear this out [24, 45, 49-52], and as in [25, 27] we adopt

$$
\sigma_{\mathrm{IGM}}=\frac{0.2}{\sqrt{z}} D M_{\mathrm{IGM}} .
$$

While the primary contribution to the density fluctuation comes from dark matter halos that are overdense in baryons, minor fluctuations due to contributions from large-scale structures such as Lyman-alpha clouds, galaxy filaments, voids, sheets and/or cosmic webs are expected to be sub-dominant with $<$ few $\times 10 \mathrm{pc} \mathrm{cm}^{-3}$ and can be ignored practically [36, 52-54].

Having established the models to be used for the main contributions to DM in Eq. (19), we show the various simulated distributions in Fig. 6 and Fig. 7.

Figure 6 gives the FRB redshift distributions for the three models used (top left panel) in terms of the MC realizations (bars) and exact forms (dashed curves). The MC realized DM distributions (top right panel) shows the total observed extraGalactic DM, denoted $D M_{\mathrm{ex}}$, with the MW contribution subtracted out through observation of the FRB direction as we will assume for the rest of the article. The difference between the solid and light, dashed bars is the difference between FRB in a universe with reionization at $z_{r}=3$ vs $z_{r}=6$ (similar to Fig. 3 ), so this gives a visual indication of how FRB abundances as a function of DM, without knowledge of redshift, can distinguish these cases. We return to this in more detail in Sec. VII. Note the nonevolving and constant with redshift source distributions have the greatest numbers of FRB at $z>3$, and so should have the greatest distinguishing power between reionization scenarios, as can also be seen by the DM distribution (top right panel). Our choice of the SFR source distribution model as fiducial is the most conservative.

The bottom panels of Figure 6 illustrate the role of the FRB host galaxy DM contribution distributions. The three models used (bottom left panel) are given in terms of the MC realizations (bars) and exact forms (dashed curves). Note the $\log$ scale for $D M_{\text {host }}$. The total observed extraGalactic DM has its distribution plotted for the three $D M_{\text {host }}$ models in the bottom right panel. Again the difference between the $z_{r}=3$ and $z_{r}=6$ reionization cases is shown by the solid and light dashed bars.

Figure 7 gives the actual MC realized DM vs redshift relations $\left(N_{\mathrm{FRB}}=1000\right.$ sources plotted $)$ for the three redshift distribution models (left panel) and the three host galaxy contribution models (right panel). The curves have been offset vertically for clarity. One can see that the SFR model distribution is weighted toward lower redshifts, the NE model toward middle redshifts, 

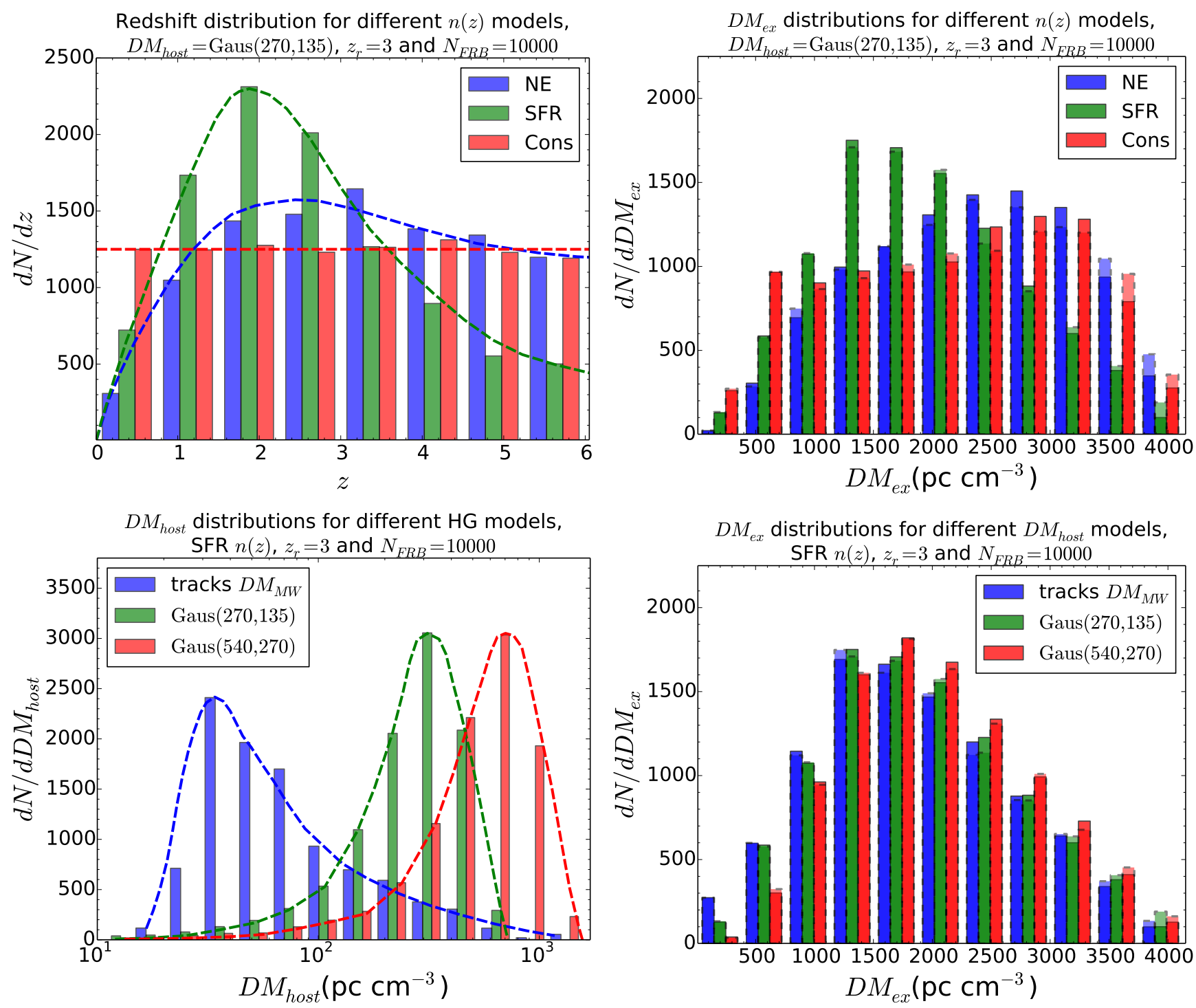

FIG. 6. Top row: [Left panel] The three different FRB redshift distribution models $d n / d z$ used (dashed lines) and their Monte Carlo realizations (solid bars). [Right panel] The effect of these different $d n / d z$ models on $d n / d D M$. These use the baseline Gaussian model of the host+local DM contribution. Lighter, dashed bars show the case if $z_{r} \geq 6$, i.e. HeII reionization outside the observed redshift range. Bottom row: [Left panel] The three different host galaxy+local DM models used (dashed lines) and their Monte Carlo realizations (solid bars). [Right panel] The effect of these different $D M_{\text {host }}$ models on the FRB distribution $d n / d D M$, including the IGM contribution. These use the baseline SFR model of $d n / d z$ (and so the green bars in the top right and bottom right panels are the same).

and the Cons model is evenly distributed. For the host galaxy contributions (all shown for the SFR redshift distribution model), the dispersion is noticeably greater at low redshifts for the high model, but at high redshift the IGM contribution dominates and little difference is seen between host models. The black vs red-brown solid curves give the expected relation from the mean contributions for universes with reionization at $z_{r}=6$ vs $z_{r}=3$, respectively.

\section{MONTE CARLO SIMULATION RESULTS USING $d n / d z$}

We compare Monte Carlo simulation data for a model with a certain FRB redshift distribution, $D M_{\text {host }}$ distribution, and cosmology including HeII reionization history, to a theory that has a different $f_{e}(z)$ history to assess the ability to probe the helium reionization epoch.

In the case where uncertainties are Gaussian this can 

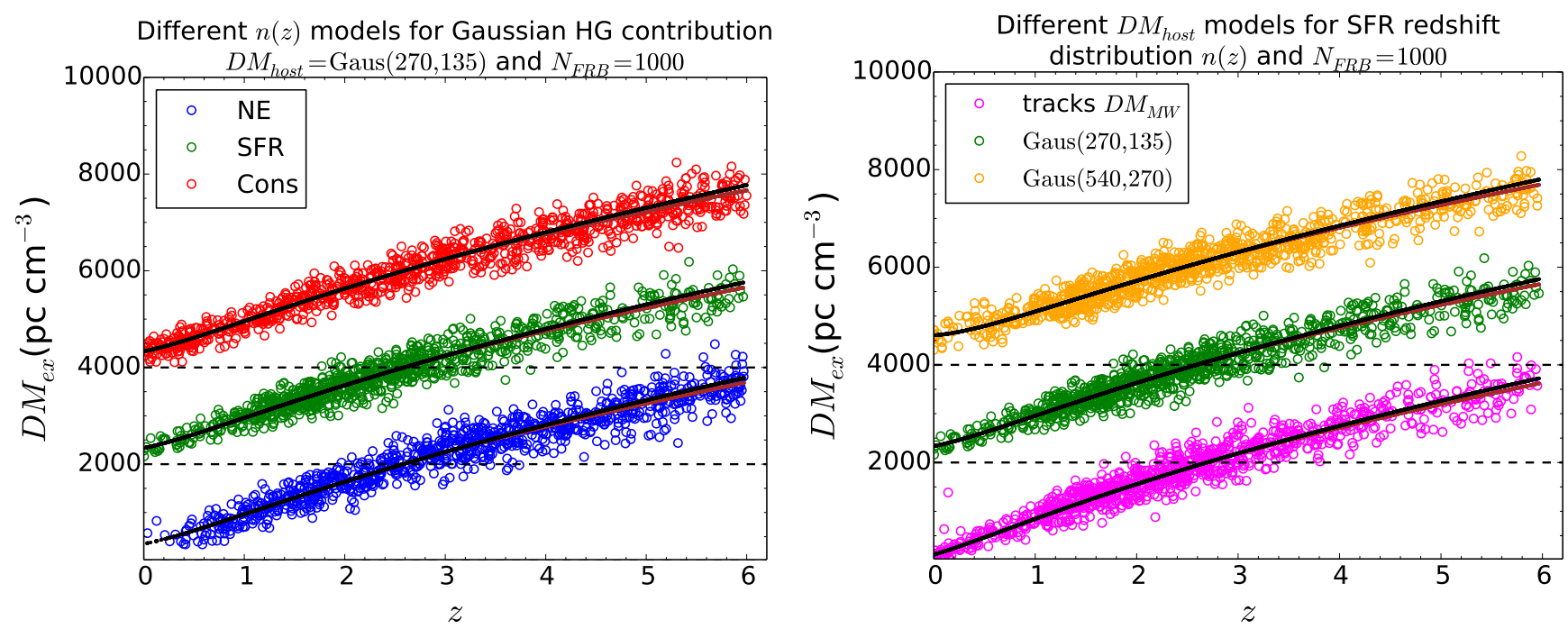

FIG. 7. Monte Carlo realizations of $1000 \mathrm{FRB}$ according to the different $d n / d z$ (left panel) and different $D M_{\text {host }}$ (right panel) models. The colored circles correspond to FRB from simulations with HeII reionisation occurring at $z_{r}=3$, with the red-brown curve corresponding to the expected mean $D M_{\mathrm{ex}}$ relation. The black curve gives the expected mean relation for $z_{r}=6$. Some models have been shifted up 2000 or $4000 \mathrm{pc} \mathrm{cm}^{-3}$ for presentation clarity; also note $D M_{\text {ex }}$ includes the host contribution and so is not zero at $z=0$.

be done through $\chi^{2}$, with

$$
\chi^{2}=\sum_{N_{F R B}} \frac{\left(D M_{\text {data }}-D M_{\text {theory }}\right)^{2}}{\sigma_{I G M}^{2}+\sigma_{C G M}^{2}+\left[\sigma_{\text {host }} /(1+z)\right]^{2}} .
$$

Again these DM refer to the extraGalactic part with the Milky Way contribution corrected for. For large $N_{\mathrm{FRB}}$ the realization scatter should diminish and the uncertainties such as $\sigma\left(z_{r}\right)$ scale as $N_{\mathrm{FRB}}^{-1 / 2}$. For smaller $N_{\mathrm{FRB}}$ the Monte Carlo nature can put more or fewer FRB at high redshift where they have leverage on determining $z_{r}$, and of course statistical fluctuations in the IGM and host contributions can also shift the results somewhat.

When looking for discrimination between cosmologies, it is useful to compare the $\chi^{2}$ they have with the data, to find the degree to which one is favored over the other, and a measure of $\sigma\left(z_{r}\right)$. Thus we use

$$
\Delta \chi^{2}=\chi_{\text {theoryx }}^{2}-\chi_{\text {theory } 1}^{2},
$$

to determine the significance of the results when comparing various models and estimating confidence intervals for measuring $z_{r}$ from FRB data.

Figure 8 shows the $\Delta \chi^{2}$ between a theory with reionization at $z_{r}$ and simulated data generated with HeIIreionization at redshift of 3 . The top panels shows how the constraint changes as we vary the FRB redshift distribution model (top left panel) and the host galaxy contribution model (top right panel). As expected, our baseline SFR redshift distribution gives the most conservative constraints as it has the fewest FRB at the high redshifts where the greatest discriminating power lies. Nevertheless it shows a convincing $\Delta \chi^{2}=107$ distinction between $z_{r}=3$ and no reionization (for $N_{\mathrm{FRB}}=10000$, with
$1 / N_{\text {FRB }}$ scaling expected). Increasing leverage comes from the NE model and greatest from the Cons model; from Fig. 6 we see that these respectively have increasing numbers of FRB at the highest redshift, where the distinction between $z_{r}=3$ and $z_{r}=6$, say, is strongest.

As the theory reionization redshift approaches $z_{r}=6$, the $\chi^{2}$ curves flatten since there are relatively fewer FRB to aid in discrimination. Conversely, for a low theory reionization redshift there is an increased lever arm and the $\chi^{2}$ curves steepen. We see much less variation among the $D M_{\text {host }}$ models since this contribution is subdominant to that from the IGM. The curves separate somewhat more for low reionization redshift as the IGM contribution is less for those models and the host contribution is relatively more, due to its $1 /(1+z)$ factor. As expected, increasing the standard deviation lowers the $\chi^{2}$.

The bottom panels of Figure 8 zoom in to show the region around $z_{r}=3$, and indicate the $\chi^{2}=1,4,9$ (i.e. $1,2,3 \sigma)$ values. We see that the effective $\sigma\left(z_{r}\right) \approx$ 0.12 . This will depend on the number of FRB, $N_{\mathrm{FRB}}$, and despite realization scatter we find that for $N_{\mathrm{FRB}} \gtrsim 1000$, the expected square root scaling $\sigma\left(z_{r}\right) \propto 1 / \sqrt{N_{\mathrm{FRB}}}$ does hold rather well, with all other parameters fixed.

We also investigate relaxing the assumption of sudden reionization. Taking a linear evolution in redshift for the ionization fraction between $z_{r, \min }$ and $z_{r \text {,max }}$ (see Appendix A), we compare in Figure 9 the sudden reionization cases (solid curves) to the gradual reionization cases (dashed curves) with the same mean $z_{r}$. They have substantially similar constraints on the reionization redshift, whether the reionization is sudden, or over a span $\Delta z=1$ or longer. The $\Delta \chi^{2}$ between sudden and gradual 

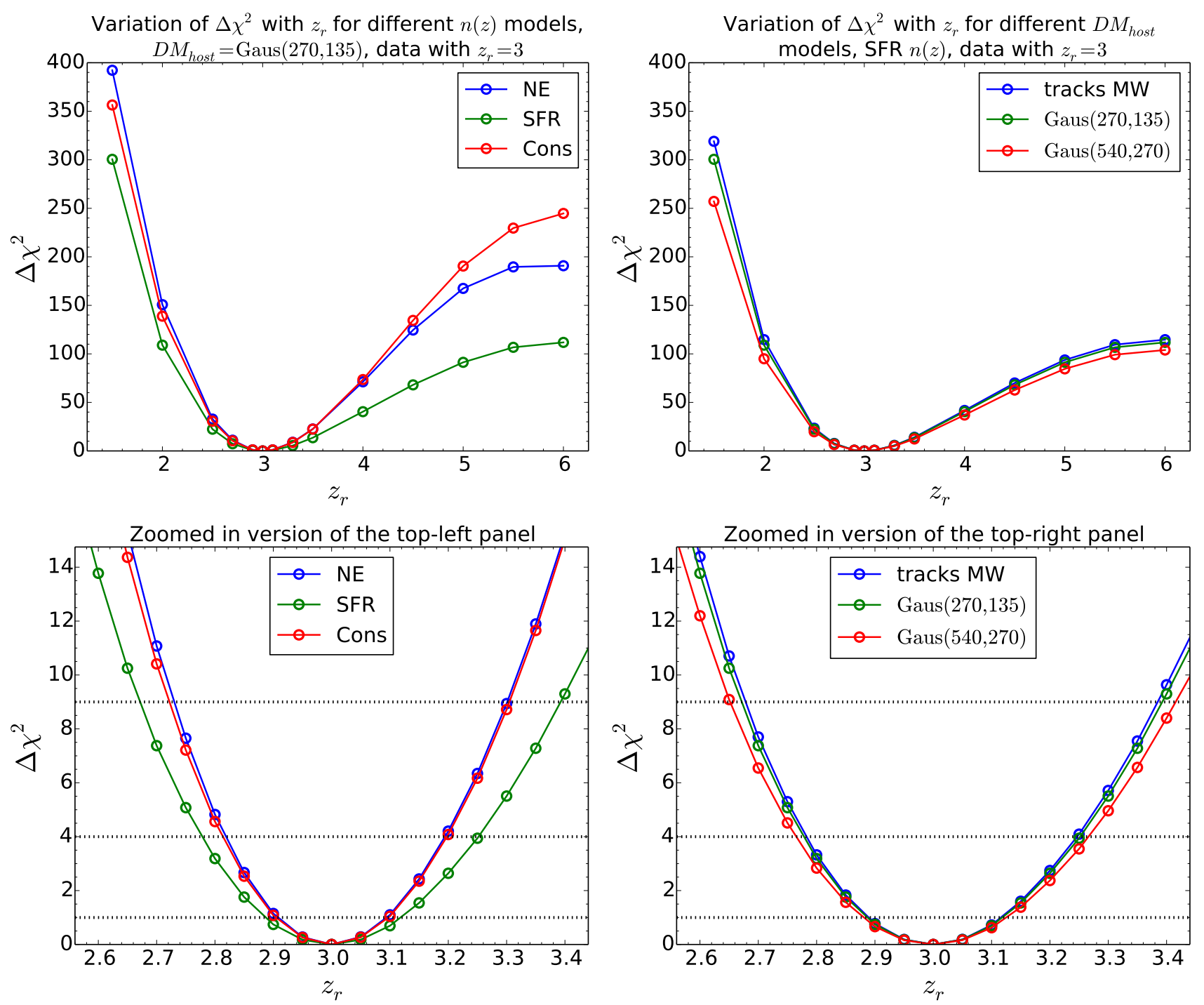

FIG. 8. Discrimination between Monte Carlo simulation data of 10000 FRB with reionization occurring at $z=3$ and a theory with reionization at $z_{r}$ is shown as a function of $z_{r}$. [Top left] Our baseline model where the FRB source distribution follows the SFR is the most conservative when compared to other $n(z)$ models. The more FRB above the reionization redshift, the greater is the constraining power. [Top right] Host galaxy plus near source models for contributions to DM make little difference, being subdominant to the IGM contribution. [Bottom panels] Zoom ins of the respective top panels showing the $1,2,3 \sigma$ constraints on reionization redshift. These numbers will scale approximately as $1 / \sqrt{N_{\text {FRB }}}$.

reionization curves for the same mean $z_{r}$ is $\lesssim 1$ for discriminating between $z_{r \text {,mean }}$ and no reionization $\left(z_{r} \geq 6\right)$ for $z_{r, \text { mean }}=3.5$ and a width $\Delta z=1$, and $\lesssim 3$ for that discrimination with $z_{r, \text { mean }}=4.5$ and a width of $\Delta z=3$.

This is a positive outcome in the sense that detection and estimation of reionization is robust to the assumption about suddenness, but does indicate that it will be difficult to distinguish the next level of detail: the duration of the reionization process.

Note also that there is rapidly reduced leverage as $z_{r, \text { mean }}$ approaches the top of the data redshift range at $z=6$, with $\Delta \chi^{2}$ falling precipitously. As we raise $z_{r}$, the $\Delta \chi^{2}=107$ discrimination between $z_{r}=3$ and no reionization reduces by roughly a factor two with every 0.5 increase in $z_{r, \text { mean }}$ (assuming $N_{\mathrm{FRB}}=10000$, and scaling linearly for smaller numbers).

We should also check that holding other parameters fixed does not unduly distort the conclusions. At these high redshifts, one expects the major influence on the Hubble parameter to be the matter density $\Omega_{m}$. Therefore we assess whether a change in $\Omega_{m}$ can mock up a change in reionization redshift. Purely comparing theoretical models without adding realization scatter, we find that while the difference in $\chi^{2}$ between the $z_{r}=3$ and $z_{r}=6$ cases with the same $\Omega_{m}$ is $\sim 107$, the difference in $\chi^{2}$ between these cases when we use $\Omega_{m}=0.315$ for the $z_{r}=3$ data but increase $\Omega_{m}$ by 0.01 to 0.325 for the $z_{r}=6$ theory is $\sim 48$. This is the minimum $\Delta \chi^{2}$ : 


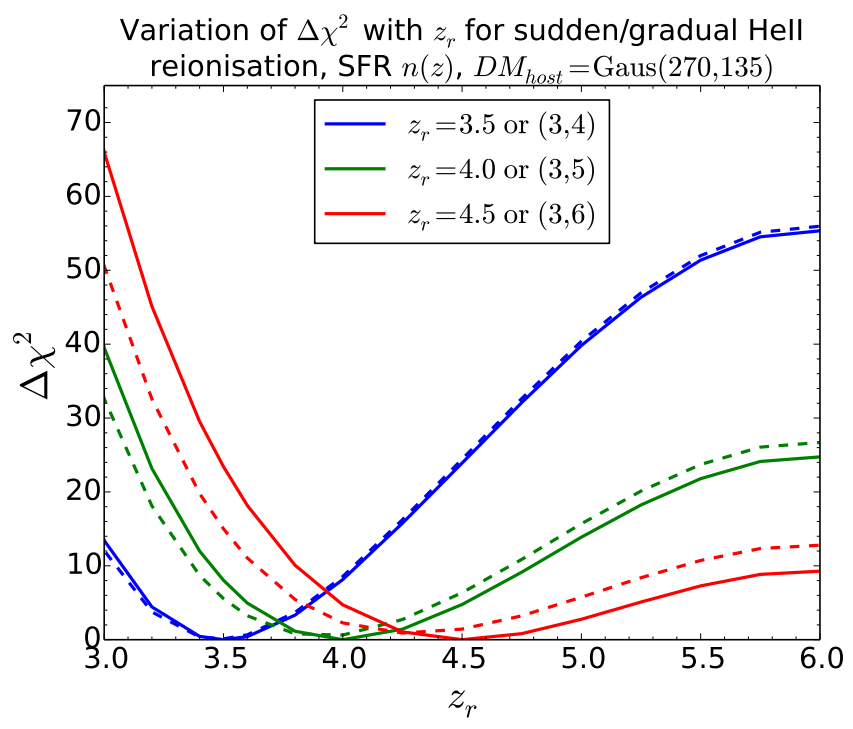

FIG. 9. Discrimination between Monte Carlo simulation data of $10000 \mathrm{FRB}$ with reionization occurring at various $z_{r, \text { mean }}$ and a theory with reionization at $z_{r}$ is shown as a function of $z_{r}$. As $z_{r, \text { mean }}$ moves to higher redshift (near the end of the data range), the detection significance of reionization occurring, i.e. $z_{r}<6$, weakens dramatically. The difference between results for sudden reionization (solid curves, labeled with $z_{r, \text { mean }}$ ) and gradual reionization (dashed curves, labeled with $\left(z_{r, \min }, z_{r, \max }\right)$, agreeing on $\left.z_{r, \text { mean }}\right)$ is generally not significant.

increasing or decreasing $\Omega_{m}$ further raises $\Delta \chi^{2}$. Thus we would still distinguish $z_{r}=3$ from $z_{r}=6$ when we simultaneously vary $\Omega_{m}$, but with looser $\sigma\left(z_{r}\right)$ due to the covariance.

When holding $z_{r}$ fixed, small changes in $\Omega_{m}$ produce $\Delta \chi^{2} \sim 70\left(\Delta \Omega_{m} / 0.01\right)^{2}$. To a large extent this is due to $\Omega_{m}$ changing DM at all redshifts, not just those above the reionization redshift. In a Fisher information analysis like that of [27], the correlation coefficient between $\Omega_{m}$ and $z_{r}$ is $r \approx 0.85$, and fixed $\Omega_{m}$ gives approximately twice as tight constraints $\sigma\left(z_{r}\right)$ as marginalizing over $\Omega_{m}$.

Figure 10 shows the covariance and degeneracy direction from the Fisher information analysis. The size of the confidence contour plotted is unimportant (it comes from [27]) but the covariance (narrowness of the ellipse) and degeneracy direction are. As mentioned above, the extent of $z_{r}$ (its uncertainty) if we fix $\Omega_{m}$ (cut along the vertical blue dashed line) is about half that if we instead marginalize. The degeneracy - the ability to trade off a shift in $\Omega_{m}$ for a shift in $z_{r}$ (shown by the dotted red line along the major axis of the ellipse) is given by

$$
z_{r}=3.0+28 \frac{\Omega_{m}-0.315}{0.315} .
$$

That is, changing $\Omega_{m}$ gives 9 times as large a fractional change in $z_{r}$, e.g. $1 \%$ shift in $\Omega_{m}$ matches with a $9 \%$ shift in $z_{r}$.

However, moving along the degeneracy direction still

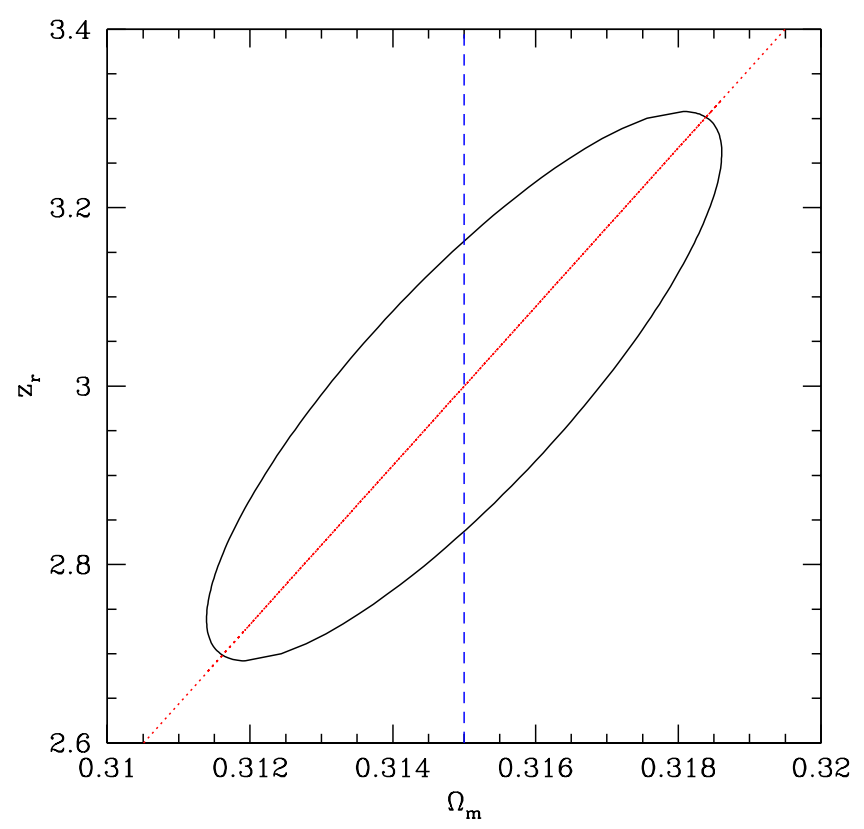

FIG. 10. Covariance between matter density $\Omega_{m}$ and reionization redshift $z_{r}$ increases the uncertainty $\sigma\left(z_{r}\right)$ by about a factor of 2 (fixing $\Omega_{m}$ corresponds to cutting across the contour vertically at the fiducial value of $\Omega_{m}$ : the vertical blue dashed line). The degeneracy direction (diagonal red dotted line) is such that a small increase in $\Omega_{m}$ acts like a large increase in $z_{r}$. The size of the contour here is unimportant, only its ellipticity (covariance) and degeneracy direction.

gives a worse fit (higher $\chi^{2}$ ), albeit more slowly than shifting in other parameter directions. Thus we would not confuse a universe with $\Omega_{m}=0.325, z_{r}=6$, say, exactly with one that had $\Omega_{m}=0.315, z_{r}=3$; the former would still be disfavored relative to the true cosmology, just not with as high significance as a change of $z_{r}$ or $\Omega_{m}$ alone (as mentioned above, $\Delta \chi^{2}=48$ rather than 107). Finally, note that Fisher information analysis is valid only for small deviations, so Eq. (23) is only good for shifts much smaller than between $z_{r}=3$ and $z_{r}=6$.

\section{MONTE CARLO SIMULATION RESULTS USING $d n / d D M$}

We now return to the FRB abundances in terms of the $d n / d D M$ distribution, which is all we could use if we lacked redshift information for the FRB. The Monte Carlo simulation data provides $d n / d D M$ as used in the probabilistic method of Sec. III.

If we had real data, we could compare it to simulations to determine which underlying cosmology, i.e. value of reionization redshift $z_{r}$, gives the best fit. Instead we compare simulations for different HeII reionization histories. For a simulated "data" sample in a universe with $z_{r}=3$ and a simulated "theory" sample with reionization 

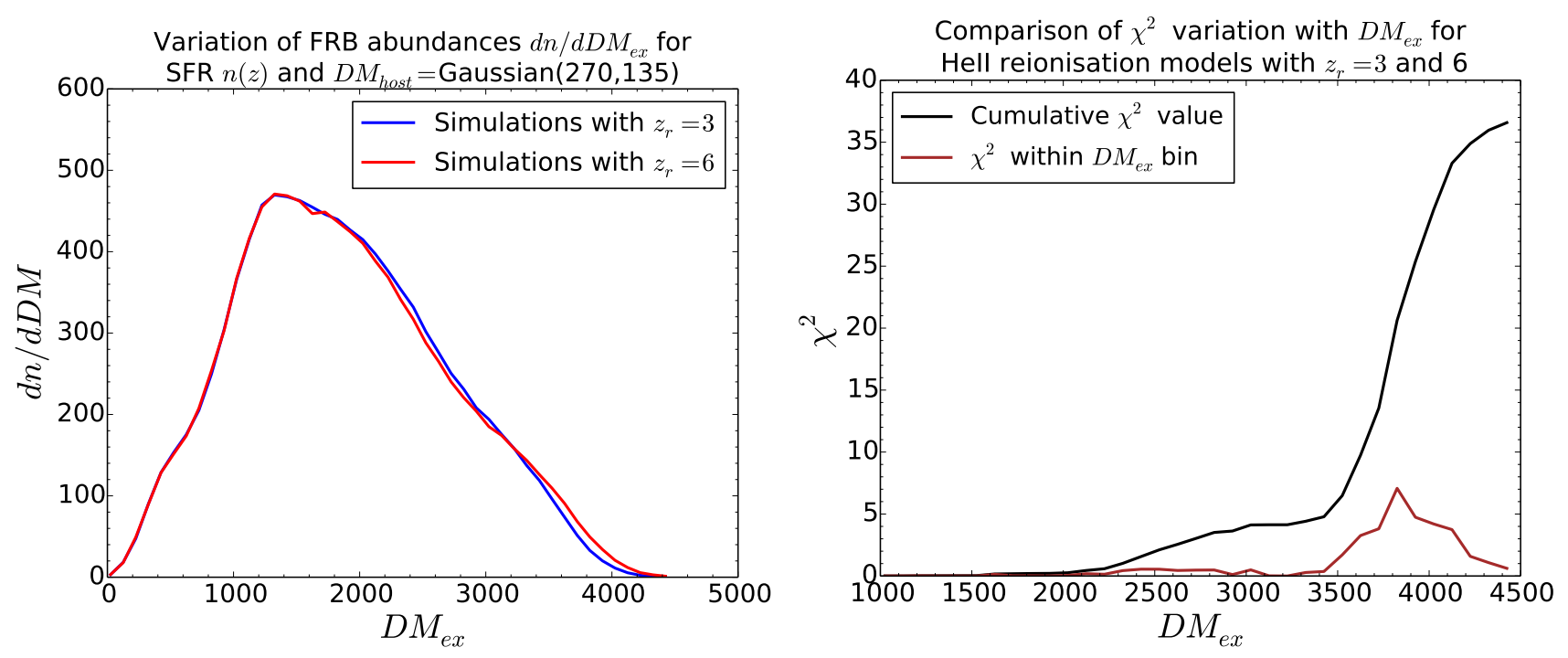

FIG. 11. FRB abundances $d n / d D M$ even in the absence of source redshift knowledge contain information on the redshift of helium reionization. [Left panel] Abundances from simulations with $z_{r}=3$ (blue) and $z_{r} \geq 6$ (i.e. outside the data range; red), are plotted vs DM. We fix $N_{\mathrm{FRB}}=10000$. [Right panel] The $\chi^{2}$ distinction between these cosmologies is shown per DM bin (lower, brown curve) and cumulative (upper, black curve).

above the observed redshift range, we compute

$$
\chi^{2}=\sum_{D M \text { bins }} \frac{\left[\frac{d n(D M)}{d D M_{\mathrm{sim} 1}}-\frac{d n(D M)}{d D M_{\mathrm{sim} 2}}\right]^{2}}{\sigma^{2}\left(\frac{d n}{d D M}\right)} .
$$

Here $\sigma^{2}(d n / d D M)$ is the variance of the data simulation results, evaluated over 100 simulations.

Figure 11 shows the results. The left panel presents the FRB abundance distributions in bins of $\triangle D M=$ $100 \mathrm{pc} \mathrm{cm}{ }^{-3}$ for the cases of $z_{r}=3$ and $z_{r} \geq 6$ (cf. Fig. 6, top right panel for a coarser view). Since the total number of FRB for $z<6$ is kept fixed, the enhancement in numbers at high DM due to very early reionization (greater ionization fraction and hence higher DM for a given $z$ ) compared to the $z_{r}=3$ case, must be compensated by fewer FRB than the $z_{r}=3$ case at intermediate $\mathrm{DM}^{2}$. Below $z_{r}=3$ the abundances are the same since the source distributions and ionization fractions are the same.

Discrimination between the cases is quantified in the right panel, with the $\chi^{2}$ contribution per DM bin and the cumulative $\chi^{2}$. At DM values characteristic of $z<z_{r}$, there is little contribution to $\chi^{2}$. The distinction increases above $z_{r}$, then levels off as DM approaches the crossing point where the abundances match due to the integral constraint on total FRB numbers, then increases again, significantly, at higher DM. The total $\chi^{2}=37$.

\footnotetext{
2 This does not happen in Fig. 3 because there the number of FRB is held fixed over all redshifts, but only FRB with $z<5$ were used in the calculations, as an observational selection cut.
}

While this is about a factor 3 lower than when we used FRB redshift information, this still allows clear discrimination between helium reionization occurring at $z_{r}=3$ and not at all within the data redshift range. Thus an ensemble of FRB with only DM observed - no redshifts - can provide important constraints (though of course the smaller sample of FRB with measured redshifts can give further probative power, and be used to "train" the DM-only sample; see the discussion in the next section).

\section{CONCLUSIONS}

Fast radio bursts are a remarkable tool for probing the ionization state of the universe at high redshift. In particular, they could be very useful for detection and characterization of HeII-reionization at $z \approx 3$. We have investigated in this work how this can be revealed through the full data set of forthcoming FRB measurements, through use of ensemble statistics rather than individual dispersion measures. This is especially relevant since most FRBs are unlikely to have follow up optical observations to identify their host galaxies and measure their redshifts.

We describe an approach based on the DM distribution, basically their abundance as a function of DM, and relate it to the redshift distribution through a probabilistic approach mimicking what is done to connect photometric estimates of redshift in optical astronomy to true redshift (or other proxy measurements vs true characteristics). While ideally the mapping would come from a training set where both DM and redshift were known, here we demonstrated the usefulness of this approach, initially with a simple Gaussian dispersion and then with 
Monte Carlo simulation.

Abundance distributions were analyzed in terms of both $d n / d z$ and $d n / d D M$ through Monte Carlo simulations. We studied the effect of several models for the FRB-host plus near source contributions to DM, and for the FRB redshift distribution. Our baseline results take the most conservative of the source models and find that the redshift of helium reionization can be determined to an uncertainty of $\sim 0.1$ in redshift, but this weakens if the reionization occurs at higher redshift. The results are found to be robust whether the HeII reionization is sudden or gradual, but the duration of reionization is quite difficult to determine. The contributions of FRB-host galaxies to the DM have relatively little effect on the determination of ionization redshift, even over a broad range of host-DM models, because at the relevant high redshifts the IGM makes much larger contributions to the DM and its fluctuations.

When one can only use the ensemble distribution in terms of DM, without FRB redshift information, the constraints on helium reionization weaken, with detection of reionization - i.e. discrimination between $z_{r}=3$ and no reionization within the observed source range - reduced by about a factor 3 from $\Delta \chi^{2} \approx 107$ to $\sim 37$, which is still $\mathrm{a} \sim 6 \sigma$ result for a sample of $10^{4}$ FRBs.

It will be interesting to pursue these ideas further, in particular the use of a training set of high redshift FRBs with known redshifts to map out the DM abundance distribution (see, e.g., [21, 55, 56]). In the optical analog, the proxy-truth relation involves not only a Gaussian dispersion but a potential mean bias and outliers. One example of greater complexity is correlated fluctuations in the IGM, where FRB DM clustering statistics (see, e.g., [57-60]) may carry information; another is if certain types of host galaxies (e.g. spiral disks), or specific near source environments, have long tails to high DM that could influence the observed DM value of even high redshift FRBs and offset the mean from the median.

With the impending explosive growth in the number of FRB, as well as redshift measurement for a small subset of these bursts, the potential to map the history of the intergalactic medium could enter a new era, with quantitative knowledge of the ionization state, redshift of helium reionization, and the use of statistical techniques such as clustering correlations to reveal energetic processes in the early universe.

\section{ACKNOWLEDGMENTS}

We gratefully acknowledge helpful discussions with Matt McQuinn, Xiangcheng Ma, and Eliot Quataert. EL thanks PK for wonderful hospitality at the University of Texas, Austin. EL is supported in part by the U.S. Department of Energy, Office of Science, Office of High Energy Physics, under contract no. DE-AC02-05CH11231 and by the Energetic Cosmos Laboratory. PK has been supported in part by an NSF grant AST-2009619.

\section{Appendix A: Effect of HeII reionisation on DM}

In this Appendix we give a brief review of the effect of HeII reionization on DM.

HeII reionisation affects $D M_{\mathrm{IGM}}$ by changing the electron density. Writing

$$
D M_{\mathrm{IGM}}(z)=K_{\mathrm{IGM}} \int_{0}^{z} \frac{\left(1+z^{\prime}\right) x\left(z^{\prime}\right)}{H\left(z^{\prime}\right) / H_{0}} d z^{\prime},
$$

where $K_{\text {IGM }}=\left(3 c H_{0} \Omega_{b} / 8 \pi G m_{p}\right) f_{I G M}=775.8 \mathrm{pc} \mathrm{cm}^{-3}$ with $f_{\mathrm{IGM}}=0.83, H\left(z^{\prime}\right)=H_{0} \sqrt{\Omega_{m}\left(1+z^{\prime}\right)^{3}+\Omega_{\Lambda}}$, and the ionization fraction

$$
x(z)=(1-Y) f_{\mathrm{H}}(z)+\frac{Y}{4}\left[f_{\mathrm{HeII}}(z)+2 f_{\mathrm{HeIII}}(z)\right]
$$

depends on the HeII reionisation epoch. Here, $f$ denotes the ionisation fractions of the individual components $(\mathrm{H} / \mathrm{HeII} / \mathrm{HeIII})$. As $\mathrm{H}$ is fully ionised for $z \lesssim 6$, we set $f_{H}=1$ for the entire FRB sample. We will consider the possibilities of both sudden and gradual He II reionisation. Throughout our Monte Carlo analysis, we assume Planck (2018) cosmological parameters with Hubble constant $H_{0}=67.4 \mathrm{~km} \mathrm{~s}^{-1} \mathrm{Mpc}^{-1}$, baryon density parameter $\Omega_{b}=0.04$, matter density parameter $\Omega_{m}=0.315$, dark energy density parameter $\Omega_{\Lambda}=1-\Omega_{m}$, and helium mass fraction $Y=0.243$.

For sudden reionisation that occurs at a reionisation redshift $z_{r}$, we have

$$
\begin{aligned}
& x=0.879\left(f_{\mathrm{HeII}}=0, f_{\mathrm{HeIII}}=1\right) \text { for } z \leq z_{r}, \\
& x=0.818\left(f_{\mathrm{HeII}}=1, f_{\mathrm{HeIII}}=0\right) \text { for } z>z_{r},
\end{aligned}
$$

whereas in the case of gradual reionisation that occurs within a redshift range of $z_{r, \min }$ to $z_{r, \max }$, we take a linear ramp

$x(z)= \begin{cases}0.879, & z \leq z_{r, \min } \\ 0.879-0.061\left(\frac{z-z_{r, \min }}{z_{r, \max }-z_{r, \min }}\right), & z_{r, \min }<z \leq z_{r, \max } \\ 0.818, & z>z_{r, \max },\end{cases}$

where the ionisation fraction $f$ of HeII increases gradually from $f_{\mathrm{HeII}}\left(z_{r, \min }\right)=0$ to $f_{\mathrm{HeII}}\left(z_{r, \max }\right)=1$ (and $f_{\mathrm{HeIII}}=$ $\left.1-f_{\mathrm{HeII}}\right)$.

The IGM DM contribution (in $\mathrm{pc} \mathrm{cm}^{-3}$ ) for sudden reionisation is

$$
D M_{\mathrm{IGM}}= \begin{cases}682 \mathcal{I}(0, z), & z \leq z_{r} \\ 682 \mathcal{I}\left(0, z_{r}\right)+635 \mathcal{I}\left(z_{r}, z\right), & z>z_{r}\end{cases}
$$

where $\mathcal{I}\left(z_{a}, z_{b}\right)=\int_{z_{a}}^{z_{b}} d z^{\prime}\left(1+z^{\prime}\right) /\left[H\left(z^{\prime}\right) / H_{0}\right]$. For gradual reionisation, we correspondingly have

$$
D M_{\mathrm{IGM}}= \begin{cases} & 682 \mathcal{I}(0, z), \quad z \leq z_{r, \min } \\ & 776 \int_{z_{r, \min }}^{z} d z^{\prime} \frac{\left(1+z^{\prime}\right) x\left(z^{\prime}\right)}{H\left(z^{\prime}\right) / H_{0}} \\ & +682 \mathcal{I}\left(0, z_{r, \min }\right), \quad z_{r, \min }<z \leq z_{r, \max } \\ & 635 \mathcal{I}\left(z_{r, \max }, z\right)+682 \mathcal{I}\left(0, z_{r, \min }\right) \\ & +776 \int_{z_{r, \min }}^{z_{r, \max }} d z^{\prime} \frac{\left(1+z^{\prime}\right) x\left(z^{\prime}\right)}{H\left(z^{\prime}\right) / H_{0}}, \quad z>z_{r, \max }\end{cases}
$$


where $x(z)=0.879-0.061\left(z-z_{r, \min }\right) /\left(z_{r, \max }-z_{r, \min }\right)$.

[1] J.I. Katz, Coherent emission in fast radio bursts, Phys. Rev. D, 89, 103009 (2014) [arXiv:1309.3538]

[2] J.I. Katz, How Soft Gamma Repeaters Might Make Fast Radio Bursts, ApJ 826, 226 (2016) [arXiv:1512.04503]

[3] K. Murase, K. Kashiyama, P. Mészáros, A burst in a wind bubble and the impact on baryonic ejecta: highenergy gamma-ray flashes and afterglows from fast radio bursts and pulsar-driven supernova remnants, MNRAS 461, 1498 (2016) [arXiv:1603.08875]

[4] P. Kumar, W. Lu, M. Bhattacharya, Fast radio burst source properties and curvature radiation model, MNRAS 468, 2726 (2017) [arXiv:1703.06139]

[5] B. D. Metzger, E. Berger, B. Margalit, Millisecond Magnetar Birth Connects FRB 121102 to Superluminous Supernovae and Long-duration Gamma-Ray Bursts, ApJ 841, 14 (2017) [arXiv:1701.02370]

[6] A. M. Beloborodov, A Flaring Magnetar in FRB 121102?, ApJ 843, L26 (2017) [arXiv:1702.08644 ]

[7] J. M. Cordes, I. Wasserman, J. W. T. Hessels, T. J. W. Lazio, S. Chatterjee, R. S. Wharton, Lensing of Fast Radio Bursts by Plasma Structures in Host Galaxies, ApJ 842, 35 (2017) [arXiv:1703.06580]

[8] G. Ghisellini, N. Locatelli, Coherent curvature radiation and fast radio bursts, A \& A 613, A61 (2018) [arXiv:1708.07507]

[9] W. Lu, P. Kumar, On the radiation mechanism of repeating fast radio bursts, ApJ 865, 128 (2018) [arXiv:1710.10270]

[10] B. D. Metzger, B. Margalit, L. Sironi, Fast radio bursts as synchrotron maser emission from decelerating relativistic blast waves, MNRAS 485, 4091 (2019) [arXiv:1902.01866]

[11] W. Wang, B. Zhang, X. Chen, R. Xu, On the Time-Frequency Downward Drifting of Repeating Fast Radio Bursts, ApJ 876, L15 (2019) [arXiv:1903.03982]

[12] Z. Wadiasingh, A. Timokhin, Repeating Fast Radio Bursts from Magnetars with Low Magnetospheric Twist, ApJ 879, 4W (2019) [arXiv:1904.12036]

[13] P. Kumar, Z. Bošnjak, FRB coherent emission from decay of Alfven waves, MNRAS 494, 2385 (2020) [arXiv:2004.00644]

[14] W. Lu, P. Kumar, B. Zhang, A unified picture of Galactic and cosmological fast radio bursts, MNRAS 498, 1397 (2020) [arXiv:2005.06736]

[15] J. I. Katz, Fast radio bursts, Progress in Particle and Nuclear Physics 103, 1 (2018) [arXiv:1804.09092]

[16] McConnell, D. et al. The Australian Square Kilometre Array Pathfinder: performance of the Boolardy engineering test array, Publ. Astron. Soc. Aust. 33, e042 (2016) [arXiv:1608.00750]

[17] R. M. Shannon et al., The dispersion-brightness relation for fast radio bursts from a wide-field survey, Nature 562, 386S (2018) []

[18] CHIME/FRB Collaboration et al., Observations of fast radio bursts at frequencies down to 400 megahertz, Nature 566, 230 (2019a) [arXiv:1901.04524]

[19] CHIME/FRB Collaboration et al., A second source of repeating fast radio bursts, Nature 566, 235 (2019b) [arXiv:1901.04525]

[20] G. Hallinan et al., The DSA-2000 - A Radio Survey Cam- era, in Bulletin of the American Astronomical Society. p. 255 (2019) [arXiv:1907.07648]

[21] W. Deng, B. Zhang, Cosmological implications of Fast Radio Burst / Gamma-Ray Burst Associations, ApJL 783, L35 (2014) [arXiv:1401.0059]

[22] B. Zhou, X. Li, T. Wang, Y-Z. Fan, D-M. Wei, Fast radio bursts as a cosmic probe?, Phys. Rev. D 89, 107303 (2014) [arXiv:1401.2927]

[23] J-P. Macquart et al., Fast Transients at Cosmological Distances with the SKA, Proceedings of Advancing Astrophysics with the Square Kilometre Array [arXiv:1501.07535]

[24] A. Walters, A. Weltman, B. M. Gaensler, Y.-Z. Ma, A. Witzemann, Future Cosmological Constraints from Fast Radio Bursts, Astrophys. J. 856, 65 (2018) [arXiv:1711.11277]

[25] P. Kumar, E.V. Linder, Use of Fast Radio Burst Dispersion Measures as Distance Measures, Phys. Rev. D 100, 083533 (2019) [arXiv:1903.08175]

[26] M. Caleb, C. Flynn, B. Stappers, Constraining the era of helium reionization using fast radio bursts, MNRAS 485, 2281 (2019) [arXiv:1902.06981]

[27] E.V. Linder, Detecting Helium Reionization with Fast Radio Bursts, Phys. Rev. D 101, 103019 (2020) [arXiv:2001.11517]

[28] E. Petroff, J.W.T. Hessels, D.R. Lorimer, Fast Radio Bursts, A\&A Review 27, 4 (2019) [arXiv:1904.07947]

[29] J.M. Cordes, S. Chatterjee, Fast Radio Bursts: An Extragalactic Enigma, Ann. Rev. Astron. Astroph. 57, 417 (2019) [arXiv:1906.05878]

[30] M. McQuinn, A. Lidz, M. Zaldarriaga, L. Hernquist, P. F. Hopkins, S. Dutta, C.-A. Faucher-Giguère, He II Reionization and its Effect on the Intergalactic Medium, Astrophys. J., 694, 842 (2009) [arXiv:0807.2799]

[31] D. Syphers, S.-F. Anderson, W. Zheng, A. Meiksin, D.-P. Schneider, D.-G. York, HST/COS Observations of Thirteen New He II Quasars, Astronomical J., 143, 100 (2012) [arXiv:1202.0236]

[32] M. Bhattacharya, P. Kumar, Population modelling of FRBs from intrinsic properties, Astrophys. J., 899, 124 (2020) [arXiv:1902.10225]

[33] Y. Niino, Fast radio bursts' recipes for the distributions of dispersion measures, flux densities, and fluences, ApJ 858, 4 (2018) [arXiv:1801.06578]

[34] J-P. Macquart, R. Ekers, FRB event rate counts II - fluence, redshift and dispersion measure distributions, MNRAS 480, 4211 (2018) [arXiv:1808.00908]

[35] M. Bhattacharya, Constraining FRB progenitors from flux distribution [arXiv:1907.11992]

[36] M. McQuinn, Locating the "missing" baryons with extragalactic dispersion measure estimates, Astrophys. J. Lett., 780, L33 (2014) [arXiv:1309.4451]

[37] P. Madau, M. Dickinson, Cosmic Star Formation History, Ann. Rev. Astron. Astroph. 52, 415 (2014) [arXiv:1403.0007]

[38] J.M. Cordes, T.J.W. Lazio, NE2001.I. A New Model for the Galactic Distribution of Free Electrons and its Fluctuations, arXiv:astro-ph/0207156

[39] K. Dolag, B. M. Gaensler, A. M. Beck, M. C. Beck, 
Constraints on the distribution and energetics of fast radio bursts using cosmological hydrodynamic simulations, MNRAS 451, 4277 (2015) [arXiv:1412.4829]

[40] E. Petroff, E. D. Barr, A. Jameson, E. F. Keane, M. Bailes, M. Kramer, V. Morello, D. Tabbara, and W. van Straten, FRBCAT: The Fast Radio Burst Catalogue, Publ. Astron. Soc. Australia 33, e045 (2016) [arXiv:1601.03547] frbcat.org

[41] A. W. K. Lau, A. Mitra, M. Shafiee, G. Smoot, Constraining HeII Reionization Detection Uncertainties via Fast Radio Bursts [arXiv:2006.11072]

[42] Y. P. Yang, R. Luo, Z. Li, B. Zhang, Astrophys. J. 839, L25 (2017) [arXiv:1701.06465]

[43] J. Xu, J. L. Han, Extragalactic dispersion measures of fast radio bursts, Research in Astronomy and Astrophysics, 15, 1629 (2015) [arXiv:1504.00200]

[44] X. Ma, E. Quataert, A. Wetzel, P.-F. Hopkins, C.A. Faucher-Giguère, D. Keres, No missing photons for reionization: moderate ionizing photon escape fractions from the FIRE-2 simulations, MNRAS, 498, 2001 (2020) [arXiv:2003.05945]

[45] J.X. Prochaska and Y. Zheng, Probing Galactic halos with fast radio bursts, MNRAS 485, 648 (2019) [arXiv:1901.11051]

[46] J. Luan, P. Goldreich, Physical Constraints On Fast Radio Burst, Astrophys. J. Lett., 785, L26 (2014) [arXiv:1401.1795]

[47] L. Gutierrez, J. E. Beckman, The Galaxy-wide Distributions of Mean Electron Density in the H II Regions of M51 and NGC 4449, Astrophys. J. Lett. 710, L44 (2010) [arXiv:1001.0276]

[48] N. Pol, M. T. Lam, M. A. McLaughlin, T. J. W. Lazio, J. M. Cordes, Estimates of Fast Radio Burst Dispersion Measures from Cosmological Simulations, Astrophys. J., 886, 135 (2019) [arXiv:1903.07630]

[49] R. Takahashi, K. Ioka, A. Mori, K. Funahashi, Statistical modelling of the cosmological dispersion measure
[arXiv:2010.01560]

[50] M. Jaroszynski, FRBs: the Dispersion Measure of Host Galaxies, Acta Astronomica 70, 87 (2020) [arXiv:2008.04634]

[51] M. Jaroszynski, Fast Radio Bursts and cosmological tests, MNRAS 484, 1637 (2019) [arXiv:1812.11936]

[52] M. Shull, C. W. Danforth, The Dispersion of Fast Radio Bursts from a Structured Intergalactic Medium at Redshifts $z<1.5$, Astrophys. J. Lett., 852, L11 (2018) [arXiv:1712.01280]

[53] B. D. Smith, E. J. Hallman, J. M. Shull, B. W. O'Shea, The Nature of the Warm/Hot Intergalactic Medium. I. Numerical Methods, Convergence, and O VI Absorption, Astrophys. J. 731, 6 (2011) [arXiv:1009.0261]

[54] V. Ravi, Measuring the Circumgalactic and Intergalactic Baryon Contents with Fast Radio Bursts, Astrophys. J. 872, 1 (2019) [arXiv:1804.07291]

[55] C.R.H. Walker, Y.-Z. Ma, R.P. Breton, Constraining Redshifts of Unlocalised Fast Radio Bursts, A\&A 638, A37 (2020) [arXiv:1804.01548]

[56] J.-P. Macquart et al., A census of baryons in the Universe from localized fast radio bursts, Nature 581, 391 (2020) [arXiv:2005.13161]

[57] K.W. Masui, K. Sigurdson, Dispersion Distance and the Matter Distribution of the Universe in Dispersion Space, Phys. Rev. Lett. 115, 121301 (2015) [arXiv:1506.01704]

[58] M. Shirasaki, K. Kashiyama, N. Yoshida, Large-scale clustering as a probe of the origin and the host environment of fast radio bursts, Phys. Rev. D 95, 083012 (2017) [arXiv:1702.07085]

[59] M. Rafiei-Ravandi, K.M. Smith, K.W. Masui, Characterizing fast radio bursts through statistical crosscorrelations, Phys. Rev. D 102, 023528 (2020) [arXiv:1912.09520]

[60] J-P. Dai, J-Q. Xia, Reconstruction of Reionization History through Dispersion Measure of Fast Radio Bursts, [arXiv:2004.11276] 